\title{
Pancreatic Acinar Cell Carcinoma with Concomitant Bullous Pemphigoid
}

\author{
David Vavra, MD; Jessica Wernberg, MD; and Rohit Sharma, MD
}

A Caucasian male, aged 72 years, was found to have a $3.9 \mathrm{~cm}$ mass in the tail of the pancreas on computed tomographic imaging during workup for hematuria. A positron emission tomography scan showed a large pancreatic tail mass with extraglandular extension, nodal uptake, and concern for involvement of the left adrenal gland (Figure 1). Endoscopic ultrasound with fine needle aspiration revealed pancreatic acinar cell carcinoma (PACC). The day after the biopsy, a blanching, erythematous rash erupted across the patient's trunk and limbs (Figure 2), with scattered intact tense bullae (Figure 3). There was no rash observed intraorally or on the face/scalp. Skin biopsies were consistent with

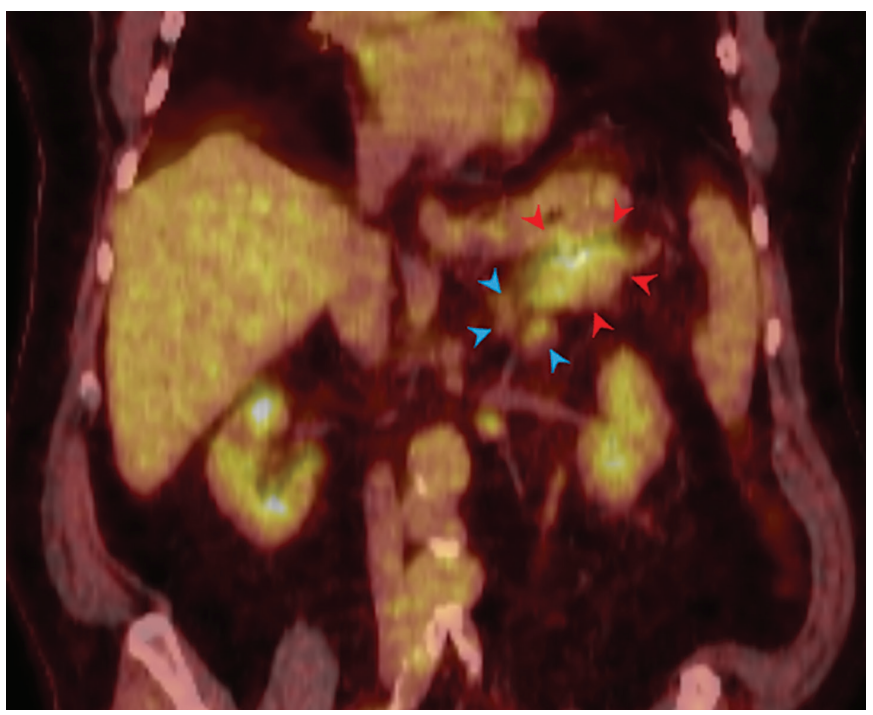

Figure 1. PET CT showing increased activity in the pancreatic tail mass (red arrows) with concern for extension into the left adrenal gland (blue arrows).

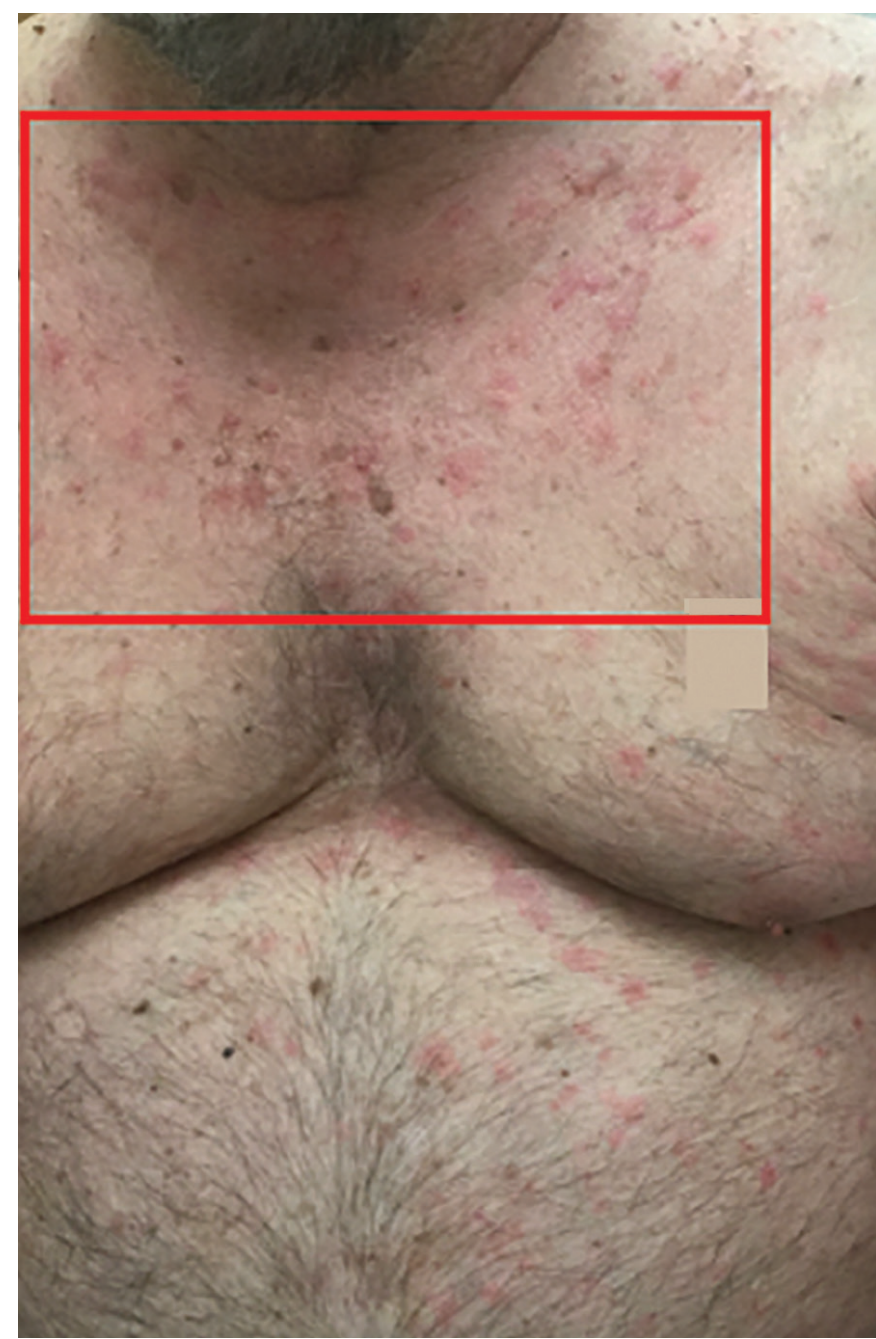

Figure 2. Blanchable, erythematous papules and plaques across the chest and abdomen (boxed in red).

Received: July I, 2020

Revised: November 21, 2020

Accepted: January 8, 2021

doi: $10.3121 / \mathrm{cmr} .2021 .1595$ 
bullous pemphigoid (BP) as evidenced by linear deposition of C3 along the epidermal-dermal junction on direct immunofluorescence testing. Treatment consisted of a 3-day steroid burst (prednisone $80 \mathrm{mg} /$ day) followed by a month long course of prednisone (40 $\mathrm{mg} /$ day), which failed to resolve the rash. The patient underwent a subtotal distal pancreatectomy, splenectomy, and en bloc left adrenalectomy; 9 of 20 regional lymph nodes had metastatic disease. The BP rash resolved by postoperative day 7, and steroids were tapered. This is the first reported case of complete resolution of BP after PACC resection.

\section{Discussion}

PACC accounts for $1 \%-2 \%$ of all pancreatic malignancies. ${ }^{1}$ Although aggressive, PACC has better survival compared to pancreatic ductal adenocarcinoma. ${ }^{1}$ Radical surgical resection for localized PACC has been recommended as the standard of care. ${ }^{1}$

Increased incidence of BP in malignancy has been suggested. Malignant disease has been reported in $5.8 \%-17.9 \%$ of patients with BP compared to $0.61 \%-5.3 \%$ in controls. ${ }^{2-4}$ Other studies have failed to confirm a significant association between these two entities. ${ }^{5,6}$ This discrepancy may be due to methodological differences between studies. Given the possible association of malignancy in those with BP, some clinicians believe an investigation for an underlying neoplasm should be undertaken in those who develop BP with particular

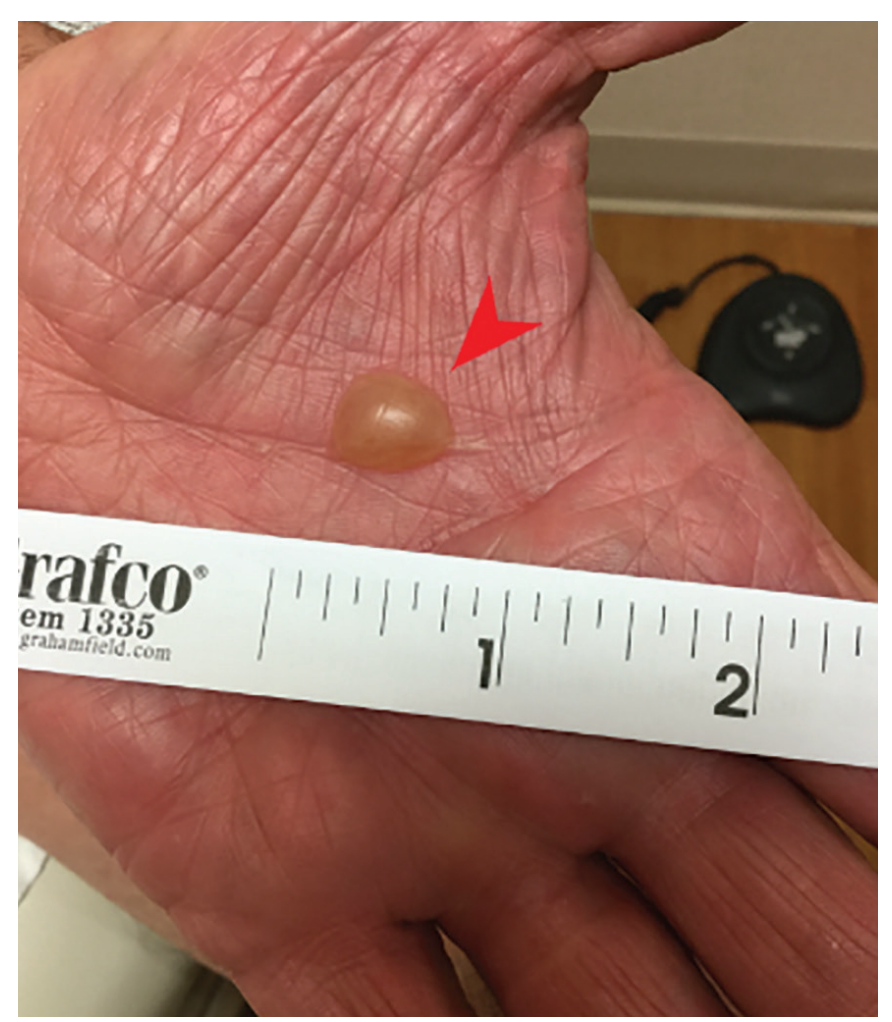

Figure 3. Left palm with a single, tense bullae (red arrow). features including: early-onset pemphigoid; those with personal history of cancer; patients with signs and symptoms suggestive of neoplasm; and BP refractory to common immunosuppressive therapy. ${ }^{7}$

A suggested mechanism of this association is the cross reaction of tumor-directed antibodies against the basement membrane zone leading to blister formation. ${ }^{7}$ Specifically, this involves laminin-332, which attaches keratinocytes to the epidermal basement membrane. ${ }^{7}$ A transmembrane glycoprotein, BP180, binds to laminin-332. Thus, the presence of an antibody against BP180 may dissociate the BP180laminin 332 complex causing disadhesion of epithelial cells. ${ }^{8}$

Varying degrees of improvement of BP have been reported after treatment of gallbladder, lung, retroperitoneum, colon, and parotid gland cancer. ${ }^{8-12}$ An analogous case report documenting complete BP resolution 7 weeks after extirpation of gastric cancer has been reported. ${ }^{13}$ In our case, BP developed the day after undergoing biopsy for PACC. An appreciably faster resolution of the rash occurred by postoperative day 7 after tumor resection in our patient. This temporal resolution is strongly suggestive of an association between BP and this patient's PACC. Further data are needed to elucidate a relationship between BP and underlying malignancy.

\section{References}

1. Schmidt CM, Matos JM, Bentrem DJ, et al. Acinar cell carcinoma of the pancreas in the United States: prognostic factors and comparison to ductal adenocarcinoma. J Gastrointest Surg. 2008;12(12):20782086.

2. Venning VA, Wojnarowska F. The association of bullous pemphigoid and malignant disease: a case control study. Br J Dermatol. 1990;123(4):439-445.

3. Chorzelski TP, Jablonska S, Maciejowska E, et al. Coexistence of malignancies with bullous pemphigoid. Arch Dermatol. 1978;114(6):964.

4. Marazza G, Pham HC, Schärer L, et al. Incidence of bullous pemphigoid and pemphigus in Switzerland: a 2-year prospective study. Br J Dermatol. 2009;161(4):861-868.

5. Lindelöf B, Islam N, Eklund G, Arfors L. Pemphigoid and cancer. Arch Dermatol. 1990;126(1):66-68.

6. Chang YT, Liu HN, Wong CK. Bullous pemphigoid--a report of 86 cases from Taiwan. Clin Exp Dermatol. 1996;21(1):20-22.

7. Balestri R, Magnano M, La Placa M, et al. Malignancies in bullous pemphigoid: A controversial association. J Dermatol. 2016;43(2):125-133.

8. Umekoji A, Tsuruta D, Inoue T, Nishimori T, Ishii M. Bullous pemphigoid as a dermadrome associated with spindle cell carcinoma of the gallbladder. J Dermatol. 2010;37(3):251-254. 
9. Takeuchi M, Okazaki A, Nakajima N, Saito Y, Nozaki M, Niibe H. Gan No Rinsho. 1986;32(5):529-533.

10. Krunic AL, Kokai D, Bacetic B, et al. Retroperitoneal round-cell liposarcoma associated with paraneoplastic pemphigus presenting as lichen planus pemphigoideslike eruption. Int J Dermatol. 1997;36(7):526-529.

11. Gilmour E, Bhushan M, Griffiths CE. Figurate erythema with bullous pemphigoid: a true paraneoplastic phenomenon? Clin Exp Dermatol. 1999;24(6):446-448.

12. Cakmak O, Seçkin D, Ceken I, Yilmaz I, Akkuzu B, Ozluoglu L. Bullous pemphigoid associated with parotid carcinoma. Otolaryngol Head Neck Surg. 2002;127(4):354-356.

13. Noguchi K, Kawamura H, Ishizu H, Okada K. Dramatic resolution of bullous pemphigoid after surgery for gastric cancer: A case report. Int J Surg Case Rep. 2014;5(4):

212-214.

\section{Author Affiliations}

David Vavra, MD; * Jessica Wernberg, MD;* and Rohit

Sharma, $M D^{*}$

*Department of General Surgery/Surgical Oncology,

Marshfield Medical Center, Marshfield, Wisconsin, USA 